\title{
Tutorial: Medical Image Processing with MITK Introduction and new Developments
}

\author{
Caspar Goch, Jasmin Metzger, Marco Nolden \\ Department for Medical and Biological Informatics, DKFZ Heidelberg, Germany \\ c.goch@dkfz.de
}

The Medical Imaging Interaction Toolkit (MITK) is a powerful tool for image processing and application development with a long history [1, 2]. It provides an easy-to-use superbuild for a variety of development scenarios and a host of customization options. This very exibility can be intimidating to new users and even more experienced users might miss out on new developments due to the sheer size of the code base. In this tutorial we will give an overview on MITK, the underlying concepts and the usage of the MITK Workbench. Afterwards we will show the attendees how to start their own software development with MITK and what pitfalls to look out for. Due to time constraints this will be in the form of a development demonstration, instead of a hands-on session. This will be followed by an introduction into more advanced usage scenarios, intended to provide a customized Workbench for special work ows or to conform to branding requirements. We will close with newly finished and ongoing developments and the new opportunities which arise based on them.

\section{Literaturverzeichnis}

1. Wolf I, Vetter M, Wegner I, et al. The medical imaging interaction toolkit. Med Image Anal. 2005;9(6):594-604.

2. Nolden M, Zelzer S, Seitel A, et al. The medical imaging interaction toolkit: challenges and advances. Int J Comput Assist Radiol Surg. 2013;8(4):607-20. 